\title{
Power Usage Effectiveness Metrics to Measure Efficiency and Performance of Data Centers
}

\author{
Mueen Uddin ${ }^{1, *}$, Raed Alsaqour ${ }^{2}$, Asadullah Shah ${ }^{1}$ and Tanzila Saba ${ }^{3}$ \\ ${ }^{1}$ Kulliah of Information Communication Technology, International Islamic University Malaysia, Jalan Gombak, 50728 Kuala Lumpur, \\ Malaysia \\ ${ }^{2}$ School of Computer Science, Faculty of Information Science and Technology, University Kebangsaan Malaysia, Bangi, 43600 , \\ Selangor, Malaysia \\ ${ }^{3}$ College of Computer and Information Sciences, Prince Sultan University Riyadh, Saudi Arabia
}

Received: 17 Aug. 2013, Revised: 14 Nov. 2013, Accepted: 15 Nov. 2013

Published online: 1 Sep. 2014

\begin{abstract}
Intensifying computation demand from enterprises has driven the growth of large, multifaceted data centers to manage current Internet, financial, commercial, and business applications. A data center comprises thousands of servers and other equipment that require substantial amounts of power to operate. This condition results in numerous challenges for the data center industry, such as massive energy consumption, underutilization of installed equipment, emission of greenhouse gases, and effect on global warming. This paper highlights the significance of identifying metrics to determine the performance and efficiency of a data center, which can help such a facility achieve operational cost savings through proper implementation of performance-measuring metrics. This paper discusses the implementation of Power Usage Effectiveness metrics in a tier-level data center in Pakistan. The results show that the overall performance value of the facility is 3.3 , which indicates poor and inefficient operations.
\end{abstract}

Keywords: Energy efficiency; Energy-efficient data center; Green IT; Metrics; Power usage effectiveness metrics

\section{Introduction}

The fast-changing and dynamic global business environment requires enterprises and their associated tier-level data centers to be more flexible to quickly adapt and respond to market changes. Among the forces that drive these changes and are receiving more attention include increasing power consumption, global warming, and corporate responsibility toward sustainability [1]. The current status of global warming, ecological deterioration, and severity of their potential consequences explain the popularity of environmental initiatives across the world [2]. The widespread use of Information Technology (IT) in various areas of business offers convenience and benefits that has propelled businesses and societies into the global arena; however, IT is contributing tremendously to environmental problems. The increased number of computers and other IT equipment as well as their frequent replacement makes the environmental impact of IT a major concern [3]. Technology is approaching the stage of creation and outsourcing of sustainable IT businesses based on the principles of green economics. Unparalleled progress in the demand for IT facilities and outsourcing has led to the expansion of large, complex, resource-exhaustive infrastructure called server farms to support business needs [4].The data center industry has emerged as a significant corporate asset, playing a vital role in business management by serving as nerve cells of any information society and IT business [5]. These facilities assimilate and store large amounts of information and process all activities and services that require Information Communication Technology (ICT) equipment, cooling and power delivery equipment, servers and storage devices to store, process, and exchange digital information [6].

Globally, the total electrical power consumption by data centers, servers, and computers is steadily increasing [4]. This increase leads to the emission of greenhouse gases that exacerbate global warming and pose risks to environmental health. A growing awareness of the need to reverse the process of environmental degradation and shift toward sustainable business practices is emerging

\footnotetext{
*Corresponding author e-mail: mueenmalik9516@gmail.com
} 
[7]. The current global financial and energy crisis, coupled with the growing sense of urgency by citizens, has motivated businesses, governments, and nonprofit organizations around the world to incorporate green practices in their policies and activities.

The data center power density has increased to an average of $15 \%$ annually between 2000 and 2009 . Continuous accumulation of computing equipment in data center design has resulted in the need to monitor, measure, and manage the performance of these installed components to accomplish energy efficiency [8]. TMeasuring power usage has become a significant concern of all business stakeholders to meet end-user expectations [9]. $\mathrm{T}$ Numerous opportunities exist to reduce power usage in data centers as a wide range of efficiency practices are available, but the industry focuses on metrics, methods, and instruments to provide these services more effectively while measuring power consumption efficiently [10]. A performance metric is a tool used to measure and assess the capability and performance of a particular component [11]. The major problem in the data center industry is the lack of credible, appropriate, and industry-acceptable standard method to categorize installed hardware and software resources and workloads into measureable groups so that available energy efficiency metrics can be applied to calculate power use [12]. The other major obstacle to improving power efficiency is the limitation of metrics (applicable measurement methods and tools) [13,14]. Research is ongoing to investigate how IT equipment power consumption varies with computation loads, as well as develop quantitative metrics, refine metrics, and set measurement protocols for benchmarking servers [15].

The research presented in this paper focuses on the context of the current power crisis in Pakistan and global warming effects worldwide. This paper aims to identify and implement Power Usage Effectiveness (PUE) metrics as a measurement tool for data centers. This paper also discusses the implementation of PUE metrics in one of the largest tier three level data centers in Pakistan (PTCL) to measure overall performance in power consumption and achieve energy-efficient and green operations.

\section{Power Measuring Problems in Data Centers}

The main problem with a tier-level data center is that no comprehensive mechanism is available for dynamically managing and measuring power consumption to model and trace power usage and thereby achieve optimum power tradeoffs. If data center managers remain unaware of power measurement problems, they may run the risk of doubling their power costs between 2005 and 2011 [14]. If these energy costs continue to double every five years, they will substantially increase to $1,600 \%$ between 2005 and 2025 [16]. These data centers not only consume a tremendous amount of energy but are riddled with IT inefficiencies $[17,18]$. Most of data center operating costs are in the areas of power usage by IT equipment. Thus, data center managers have to understand, manage, analyze, and measure the power usage and consumption in their facilities [19]. Given rising energy costs, energy security concerns, environmental pressures, and business demands, data center operators will soon be targeted, measured, grouped or labeled based on the efficiency of their facilities [20,21].

The data center industry has no standardized metrics to determine the power costs associated with a particular infrastructure element [22]. This gap in traceability means that businesses cannot make better decisions with respect to infrastructure investment or management. Addressing data center power measurement is more important than ever [23]. The data center industry has a long way to go in raising awareness, as well as establishing and monitoring relevant metrics. This demand requires the development of green metrics with a broad emphasis on performance and measurement of related power consumption in almost all aspects of data center operation [24].These metrics would greatly improve the efficiency of a data center and provide services with high response time and reduced power consumption.

The problem with standard measurement techniques is that they are time consuming and costly to deliver good results [25]. Power measurements must be taken over a period of time long enough to incorporate the changes in power consumption caused by periodic workload fluctuations. When this factor is considered, along with the fact that data centers are constantly changing entities, measurements can be inaccurate and out-of-date by the time they have been gathered. How does an IT organization measure data center power consumption accurately enough to reflect efficiency improvements, but fast enough so that the information is timely and viable [26]. From a power standpoint, a data center is a hierarchy of electrical devices. Utility power feeds one or more Uninterruptible Power Supply (UPS), which in turn feeds a greater number of Power Distribution Units (PDUs), each of which feeds multiple racks [27]. Measuring power consumption lower in the hierarchy provides more accurate measurements but takes longer to obtain results. More devices measured make the measurement more expensive and the target more dynamic [7]. Only one power meter is used for the facility relative to tens of PDUs and thousands of servers. Measured electricity usage can be obtained from utility bills, meters, or facility management software tools [28]. Sources for estimating power consumption on a device or component level are vendor-supplied information, including equipment faceplates and site planning guides. Other sources for determining energy usage are power meters and analyzers, as well as power distribution devices that can also measure and report power usage [29]. 


\section{Problem Statement}

Data centers use thousands of servers and other IT equipment to perform processing for end users to facilitate and accomplish business goals. As businesses, including e-businesses, grow rapidly, large and complex data centers become essential. In most data centers, almost $90 \%$ of the IT equipment are idle most of the time but consume large amounts of power and simultaneously generate an enormous amount of $\mathrm{CO} 2$, which is hazardous to environmental sustainability and contributes to global warming. The problem with the data industry is that no proper standardized mechanism or credible metric is available to measure the power consumption of different equipment.

\section{Related Work}

Power management and efficiency has become an important issue with rising power dissipation in modern hardware systems affecting overall system design [30]. This issue is relevant to data centers, where the cost of power supply and cooling plays a major role. The recent advancement in hardware designs and features focus on built-in energy management tools but also disable certain device features or reduce the speed during low utilization phases [31]. To promote energy efficiency, enterprises need benchmarks to assess their effectiveness. Unfortunately, limited attention has been given to the development of metrics or benchmarks for workloads, as well as guidelines to gauge the efficacy of energy optimization from a system perspective [32]. Efforts are under way to establish benchmarks for energy efficiency in data centers, but these efforts are incomplete. Other studies and metrics [33] have emphasized energy-delay product or performance per watt to determine the energy efficiency of processors and servers without fixing workloads.

Several researchers are working to propose solutions, such as algorithms and techniques related to workload/process allocations in data centers, and load-balancing algorithms to balance and distribute workload among server machines and thereby improve the performance of blade servers contained in racks [34]. Dynamic Voltage Scaling (DVS) techniques proposed by AlEnawy and Ayadin [35] emphasize energy savings in systems that process mission-critical real-time tasks. The solution proposed by Yuan and Nahrstedt [36]is to save power by implementing a soft real-time scheduling algorithm that focuses on small handheld devices such as mobile phones. Merkel and Bellosa [37] developed a power utilization model based on memory (RAM and ROM) to achieve efficient energy savings. The technique proposed by Mastroleon [38] directly controls hardware energy savings by adjusting power distribution to different components in the data center. The problem with these hardware-based techniques is that most of them focus on real-time tasks and do not consider minimizing energy use by active computing nodes [39]. A popular trend currently being implemented in data center architecture is the use of large-scale, modular data centers composed of shipping containers filled with servers, but more radical proposals range from micro data centers placed in condominium closets to floating barges filled with servers running off of power generated from ocean currents [40].

A technique proposed by Heath [41] focuses on measuring power consumption via different data center devices using performance of power consumed in either homogeneous or heterogeneous racks executing different applications. The demand model proposed by Adachi [42] is more applicable to the data center industry because it combines the workloads and resources of data centers into resource pools, then provides those resources based on priority or predicted algorithm use. This technique satisfies the demand by different processes retaining the optimization values set for achieving energy utilization. Petrucci [43] proposed a unified framework for large grid computing systems using different techniques such as policies for power off idle nodes, thereby predicting the future load on servers to save power. Capit [44] highlighted different aspects of developing a green IT implementation framework to build green data centers, but this proposal lacks different techniques to focus on the emission of greenhouse gases, proper recycling policy, and service-level agreements between service providers and end users. Based on these findings, this paper proposes a novel, measurement-based method to characterize and improve the power efficiency of a data center. The proposed metrics methodology provides not only a clear set of measurement-based best practices metrics, but also clear guidance for data center managers to substantially improve the efficiency and performance of their facilities. This study proposes to use PUE metrics in measuring the performance of a data center.

\section{Proposed Work}

The phenomenon of establishing green, energy-efficient data centers has become the catalyst for the emergence of Green IT, and has piqued the interest of information system researchers, business practitioners, and politicians. Green IT solutions provide environment-friendly techniques and methods toward implementing more effective and efficient organizational and national strategies, as well as policies to attain sustainable businesses worldwide.

A major gap in the current literature is the lack of appropriate standardized metrics to measure the energy efficiency of data centers [45]. To advance this line of research, this study attempts to bridge the gap by highlighting the importance of green metrics in measuring the performance of a data center, then identifying the most common metrics adopted by the industry to measure 
the energy efficiency initiatives of such centers. This study focuses on the significance of implementing and applying the most prevalent green metric, PUE, to measure the energy efficiency of a data center.

\subsection{Power Efficiency in Data Centers}

Energy efficiency refers to any behavior that results in the use of less power. It is achieved by using different techniques and methods that require less power to perform the same task. Energy efficiency in computing has historically improved more slowly than performance or cost [46]. The excessive use of power in data centers has placed unnecessary load on local utilities and caused environmental concerns for pollution and ecological stewardship [3]. These concerns are sufficiently accurate that large enterprises are considering energy efficiency as a priority and a topic of intense debate. Numerous organizations started to build their data centers near electric plants in cold weather environments [40]. Many corporations are also applying power-saving methods for their equipment and facilities under the banner of green, energy-efficient computing [52].

Power demands required by data center industry are met by considering the following factors:

- Power is available to the data center facility, but power distribution infrastructure is constrained.

- Power is available to the facility, but standby or backup power is insufficient for growth.

- Power is available in the general area, but utility constraints prevent delivery to the data center.

- Power costs are excessive in the region where the IT equipment and facilities are located.

The development and implementation of energy-efficient resource management strategies in data centers has become a prerequisite to implement energy-efficient, green, and environment-friendly data centers [47]. The actual energy consumed by a data center does not affect the cost of infrastructure, but is reflected in the electricity cost consumed by the system during the period of operations. Considering high power consumption in data centers, we believe that the major problem is how to minimize the peak power required to feed a completely utilized system.

\subsection{Energy Efficiency Metrics}

Power consumption and energy efficiency are key factors in the initial design and day-to-day management of computer systems. They have a direct relationship as energy efficiency decreases the rate of power consumption. Energy efficiency has become a significant metric that is progressively implemented to evaluate and measure the energy utilization of devices installed in data centers [48]. Many energy efficiency metrics for networking protocols and devices have been proposed, but most of them have been specialized for specific networking software or equipment and are being used in an ad hoc manner. For instance, the ones commonly used in the literature include absolute power in watts, power per bit, and normalized energy consumption (sum of energy consumed by all components) to full-duplex throughput for networking devices [49]. Metrics facilitate energy optimization by defining energy-efficient techniques to implement green, energy-efficient data centers. They must correlate strongly with the concerns of business enterprises and end users, while also being understandable, general, and practical to adopt and calculate [13]. A metric must have different proficiencies such as assessment tools, analysis and benchmarking features, design, plan, and implementation characteristics to help define and improve its performance and measurement capability [13]. These metrics identify potential opportunities to reduce energy use in data centers. The Green Grid has specified the following characteristics of efficient data center metrics [51].

- The metric name should be clear and intuitive.

- The metric should be capable of scaling according to the purpose for which it was initially created and should factor in technological, economic, and environmental changes.

- The metric must be scientifically accurate and used precisely.

- The metric must be granular enough to analyze individual aspects and provide data-driven decisions.

\subsection{Criteria for Metrics Selection}

Energy efficiency metrics and benchmarks are used to track the performance of a data center in power and energy use at different levels. These metrics identify potential opportunities to reduce energy use in data centers. Limited focus has been given to metrics and models to highlight the significance of power management and energy optimization in the data center industry [8]. The primary problem faced by any metric when applied to energy efficiency calculation is the lack of a standardized system for categorizing different components of the data center. The metric selection criteria help to better employ all equipment assets in the data center so that their utilization ratio can be maximized. The criteria also provide capability to benchmark performance values and demonstrate continuous improvement as well as superior energy efficiency to reduce the total cost of ownership of a data center. The metrics must cover certain areas for measurement, such as IT power utilization, facilities power utilization, system space used within the data center, and geographical location of the data center. According to the criteria, metrics should have the 
following properties defined as attributes for the selection to be an effective metric.

\section{Definition of metrics \\ 2. Measurement capability \\ 3. Usage of metrics}

\subsubsection{Definition of Metrics}

The Metric must provide and contain the following attributes:

- It must clarify the definition of data center performance and energy.

- It must define the area to be measured in the data center.

- It must specify the base of energy values so that new values can be compared and benchmarks can be set.

- It must define the scope of data center management according to the type of services it provides.

- It must provide solutions for energy efficiency improvement according to data center activities and infrastructure.

- It must clearly define the method of selecting IT equipment and total power as input to the data center.

\subsubsection{Measurement Capability}

- Proper regulations should be set on measurement methods to calculate the efficiency and achieve desired objectives.

- In a situation where measurement is difficult to perform and results do not show the desired values, a mechanism should be used to set some estimation methods to find the nearest values.

- Measurement conditions such as service level agreements should be followed while measuring values.

- The metric must be simple and cost-effective, that is, measurement costs should be low.

\subsubsection{Usage of Metrics}

- The metric must consider data center diversity and divide the data center into segments before metric application.

- It must follow security considerations and constraints already deployed.

- It must be easy to use and serve as motivation for both businesses and tenants (users).

- It must have provision of numerical information.

- It should provide an effective way to evaluate cooperative efforts for energy efficiency improvement activities.

\section{Implementation of PUE Metrics}

In computing the power efficiency of data centers, metrics are necessary to substantially measure this power efficiency from time to time to help top management make correct decisions toward implementing green data centers. In quantifying the power efficiency of data centers, PUE has been selected according to a criterion described and was implemented in one of the tier-level data centers in Pakistan. The criterion is defined as total power used by the data center divided by the total power consumed by the ICT equipment. This criterion provides opportunities to improve data center operational efficiency and compares efficiency with competitive data centers. The function of PUE in a data center is implemented by calculating the total power drawn from utility as the sum of total facility power for the data center, and the total power consumed by the non-data center components, as shown in Fig.1.

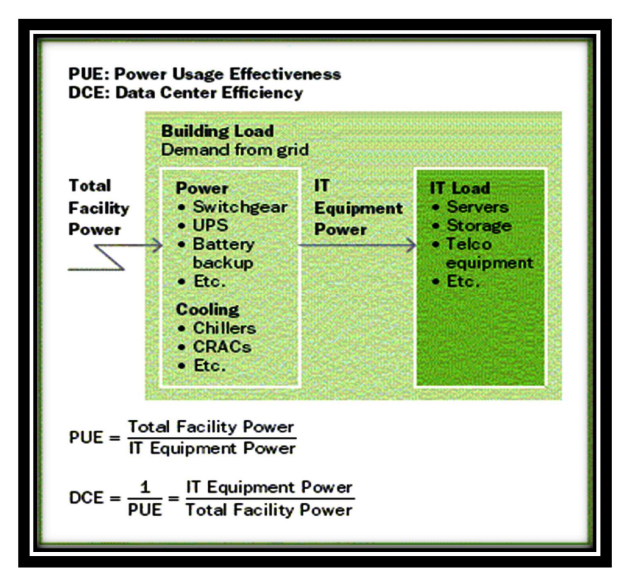

Fig. 1: Illustration of PUE Metrics

IT equipment power would be measured after all power conversion, switching, and conditioning is completed before the IT equipment itself. The most likely measurement point would be the output of computer room PDUs. This measurement should represent the total power delivered to compute the equipment racks in a data center. Table 1 presents the distribution of data center sub-systems in three main categories according to the power they consume and workloads they execute.

\section{Results and Discussions}

The rising cost of energy and corresponding increases in power consumption drive the need for server benchmarks with a broad focus on speed-oriented performance and associated power consumption. Existing 
Table 1: Data center Sub-system Distribution

\begin{tabular}{|c|c|c|c|}
\hline $\begin{array}{l}\text { IT } \\
\text { Load }\end{array}$ & List of Subsystem & $\begin{array}{l}\text { Total Physical } \\
\text { Infrastructure }\end{array}$ & $\begin{array}{l}\text { Other } \\
\text { Equipment }\end{array}$ \\
\hline$\checkmark$ & Servers & & \\
\hline$\checkmark$ & $\begin{array}{l}\text { Storage } \\
\text { Equipment }\end{array}$ & & \\
\hline$\checkmark$ & $\begin{array}{l}\text { Networking } \\
\text { Equipments }\end{array}$ & & \\
\hline$\checkmark$ & KVM Switches & & \\
\hline$\checkmark$ & $\begin{array}{l}\text { Disaster Recovery } \\
\text { IT } \quad \text { Load } \\
\text { Equipments }\end{array}$ & & \\
\hline$\checkmark$ & $\begin{array}{l}\text { Network } \\
\text { Operation } \\
\text { Equipments }\end{array}$ & & \\
\hline & UPS & $\checkmark$ & \\
\hline$\checkmark$ & Monitors & & \\
\hline$\checkmark$ & Workstations/PCs & & \\
\hline & Switch gear & $\checkmark$ & \\
\hline & Generators & $\checkmark$ & \\
\hline & $\begin{array}{l}\text { Power Distribution } \\
\text { Units }\end{array}$ & $\checkmark$ & \\
\hline & Batteries & $\checkmark$ & \\
\hline & Chillers & $\checkmark$ & \\
\hline & $\begin{array}{l}\text { Computer room air } \\
\text { conditioning units } \\
\text { (CRACs) }\end{array}$ & $\checkmark$ & \\
\hline & $\begin{array}{l}\text { Direct expansion } \\
\text { air handler (DX) } \\
\text { units }\end{array}$ & $\checkmark$ & \\
\hline & Pumps & $\checkmark$ & \\
\hline & Cooling towers & $\checkmark$ & \\
\hline & $\begin{array}{l}\text { Chilled water } \\
\text { pumps }\end{array}$ & $\checkmark$ & \\
\hline & Cooling Tower & $\checkmark$ & \\
\hline & $\begin{array}{ll}\text { Pipe } & \text { freeze } \\
\text { protection } & \end{array}$ & $\checkmark$ & \\
\hline & $\begin{array}{l}\text { Air Compressor } \\
\text { Centralized } \\
\text { humidifiers }\end{array}$ & $\begin{array}{l}\checkmark \\
\checkmark\end{array}$ & \\
\hline & Condensate pumps & $\checkmark$ & \\
\hline & Unit heaters & $\checkmark$ & \\
\hline & Dry coolers & $\checkmark$ & \\
\hline & Condensers & $\checkmark$ & \\
\hline & Well pumps & $\checkmark$ & \\
\hline & $\begin{array}{l}\text { Lights in DC } \\
\text { whitespace }\end{array}$ & $\checkmark$ & \\
\hline & $\begin{array}{l}\text { Lights in } \\
\text { mechanical and } \\
\text { electrical rooms }\end{array}$ & $\checkmark$ & \\
\hline & Outdoor lights & $\checkmark$ & $\checkmark$ \\
\hline & $\begin{array}{l}\text { Personal office } \\
\text { lights }\end{array}$ & & $\checkmark$ \\
\hline & $\begin{array}{ll}\text { Lights in } & \text { DC } \\
\text { personal area }\end{array}$ & & $\checkmark$ \\
\hline
\end{tabular}

Table 2: PUE Efficiency Values

\begin{tabular}{|l|l|l|}
\hline PUE & DCE & Level of Efficiency \\
\hline 3.0 & $33 \%$ & Very Inefficient \\
\hline 2.5 & $40 \%$ & Inefficient \\
\hline 2.0 & $50 \%$ & Average \\
\hline 1.5 & $67 \%$ & Efficient \\
\hline 1.2 & $83 \%$ & Very Efficient \\
\hline
\end{tabular}

power-measurement methodologies vary in their ability to meet this need. Maximized computational performance remains a major goal for measuring IT equipment and total facility load values. Before PUE metrics are implemented, the following steps were conducted to ensure proper implementation of these metrics in measuring energy efficiency followed by analysis to obtain aggregate values for benchmarking and standard setting

1. Selecting the appropriate metric to become an expedient and practical metric based on the aforementioned criteria.

2. Selecting the data center type (Tier I, II, III, and IV).

3. Measuring the current individual and collective power consumption or baseline values.

4. Identifying and highlighting potential areas for efficiency improvement such as IT equipment power consumption and total facility power consumption.

5. Selecting the elapsed time of assessment to measure values.

6. Calculating the mean of source load values for the assessment period.

7. Calculating the mean of values obtained by applying the proposed metric.

8. Calculating and reporting the highest day-to-day power measurement values recorded during the assessment period.

9. Calculating and reporting lowest day-to-day values recorded during the assessment period.

10. Normalizing the calculated values collected from different sources.

11. Analyzing the calculated values and setting benchmarks.

The proposed metric selection criteria along with the aforementioned methodology to select appropriate metrics and generate values help data center managers make efficiency decisions. Typical tier-level data centers must have a PUE value closer to 2 or even lower than 2 . This value can be achieved by properly using the resources and other equipment in the data center. PUE values can range from 1 to infinity. A PUE value approaching 1 would indicate $100 \%$ efficiency, which indicates that all the power is used by the IT equipment only. Table 2 shows the range of PUE values for data centers. These values show the actual efficiency of any tier-level data center [51]. 


\subsection{PUE Calculations}

PUE was calculated by employing the aforementioned methodology in a PTCL data center in Pakistan. Two values are required to calculate the exact efficiency value.

1. Total power consumed by IT equipment. 2. Total power consumed by facility.

The results obtained after applying PUE metrics are:

Total IT Equipment Power $=60 \mathrm{Kw}$ Total Facility Power $=200 \mathrm{Kw}$

PUE $=$ Total Facility Power/Total IT Equipment Power

$\mathrm{PUE}=200 / 60=3.3 \mathrm{PUE}=3.3$

The results were normalized and showed that performance and cost were the most significant attributes. These results demonstrate that managing capital costs and operating expenses are vital to data center viability. The results also show that the overall performance of the PTCL data center in energy efficiency was very poor or inefficient with a PUE value of 3.3, although PTCL was a new data center established with tier level 3 specifications.

The results highlight the need to implement green, energy-efficient data centers. Discussions were conducted with top-level management, who admitted that they were not implementing any proper framework or metric to measure the efficiency of their facilities. This lack of knowledge regarding energy efficiency was caused is related to the unawareness of top-level management about the concept of green data centers. Numerous reasons are behind this poor performance. One reason is that a data center contains approximately 150 racks, but only $15 \%$ of them are filled and performing their processing, whereas the remaining $85 \%$ only consume power without being properly utilized; thus, the overall performance was inefficient. This consumption also contributes to the emission of hazardous greenhouse gases. The other reason for this inefficiency is the lack of usage of effective technologies such as virtualization, thin provisioning, and physical to live migration, data deduplication, and other energy conservation techniques.

\subsection{Goals of PUE Metrics}

To determine the effectiveness of PUE metrics, we assessed them in a tier-level data center against the goals such as effectiveness in reporting, targets, education, analysis, and decision support from different data center managers. These managers agreed and provided the following answers as shown in Table 3.

\section{Conclusion}

Energy efficiency has emerged as one of the major design requirements for modern computing systems, such as data centers, as they continue to consume substantial amounts
Table 3: Goals of PUE Metrics

\begin{tabular}{|l|l|}
\hline Specified Goals of PUE & Results \\
\hline $\begin{array}{l}\text { 1. Provides a clear, preferably instinctive } \\
\text { understanding of measuring values. }\end{array}$ & Yes \\
\hline $\begin{array}{l}\text { 2. Measures and significant power consumption } \\
\text { values of different IT equipment and data center } \\
\text { components are clearly defined. }\end{array}$ & Yes \\
\hline $\begin{array}{l}\text { 3. Metrics are stable, persistent, and extensible as } \\
\text { the scope of power efficiency clause increases. }\end{array}$ & Yes \\
\hline $\begin{array}{l}\text { 4. Metrics highlight peak load value to point out } \\
\text { improvement areas in the design of data center } \\
\text { facilities. }\end{array}$ & Yes \\
\hline $\begin{array}{l}\text { 5. Metrics are reversible, which means that they } \\
\text { can be used to determine the power usage at the } \\
\text { electrical input to data center for any specified } \\
\text { device or equipment within the data center. }\end{array}$ & \\
\hline $\begin{array}{l}\text { 6. Metrics can support what if analysis for IT and } \\
\text { data center operators in determining the power } \\
\text { improvements and ROI. }\end{array}$ & Yes \\
\hline $\begin{array}{l}\text { 7. Metrics can provide data center managers with } \\
\text { clear understanding of the effects of change. }\end{array}$ & Yes \\
\hline
\end{tabular}

of electrical power. High operating costs incurred by computing resources and their energy usage lead to significant emissions of greenhouse gases into the environment. Unless energy efficient techniques and algorithms are developed to manage computing resources, IT contribution to global energy consumption is expected to rise rapidly. The power management problem becomes more complicated when considered at the data center level. The results obtained after applying the PUE metrics show a PUE value of 3.3, which indicate a highly inefficient data center where managers are unaware of green and energy-efficient concepts and have no knowledge of performance measurement for own facilities. The results demonstrate a strong need to implement energy efficiency techniques and methods to overcome these inefficiencies. The data center manager agrees that metrics are essential tools and PUE is a standard method to be used occasionally in measuring the performance of the data center examined in this study.

\section{Acknowledgment}

This study was partially funded by the University Kebangsaan Malaysia under Grant Nos. UKM-GUP-2012-089 and FRGS/1/2012/SG05/UKM/02/7. 


\section{References}

[1] Dao, Viet and Langella, Ian and Carbo, Jerry: From green to sustainability: Information Technology and an integrated sustainability framework, The Journal of Strategic Information Systems, North-Holland, 20, 63-79 (2011).

[2] Uddin, Mueen and Talha, Muhammad and Rahman, Azizah Abdul and Shah, Asadullah and Ahmed, Jameel, Green Information Technology (IT) framework for energy efficient data centers using virtualization, International Journal of Physical Sciences, 7, 2052-2065 (2012).

[3] Uddin, Mueen and Rahman, Azizah Abdul, Energy efficiency and low carbon enabler green IT framework for data centers considering green metrics, Renewable and Sustainable Energy Reviews, Elsevier, 16, 4078-4094 (2012).

[4] Lacity, Mary C and Khan, Shaji A and Willcocks, Leslie P, A review of the IT outsourcing literature: Insights for practice, The Journal of Strategic Information Systems, Elsevier, 18, 130-146 (2009).

[5] Daim, Tugrul and Justice, Jay and Krampits, Mark and Letts, Matthew and Subramanian, Ganesh and Thirumalai, Mukundan, Data center metrics: an energy efficiency model for information technology managers, Management of Environmental Quality: An International Journal, Emerald Group Publishing Limited, 20, 712-731 (2009).

[6] Blazek, Michele and Chong, Huimin and Loh, Woonsien and Koomey, Jonathan G: Data centers revisited: assessment of the energy impact of retrofits and technology trends in a high-density computing facility, American Society of Civil Engineers, 10, 98-104 (2004).

[7] Bianchini, Ricardo and Rajamony, Ramakrishnan: Power and energy management for server systems, IEEE, 37, 68-76 (2004).

[8] Ranganathan, Parthasarathy and Rivoire, Suzanne and Moore, Justin, Models and Metrics for Energy-Efficient Computing, Advances in Computers, Elsevier, 75, 159-233 (2009).

[9] Tung, Teresa, Data Center Energy Forecast, Silicon Valley Leadership Group, San Jose, CA, (2008).

[10] Capra, Eugenio and Merlo, Francesco, Green IT: everything starts from the software, Proceedings of the 17th European Conference on Information Systems (ECIS 2009), (2009).

[11] Wang, Lizhe and Khan, Samee U, Review of performance metrics for green data centers: a taxonomy study, The Journal of Supercomputing, Springer, 1-18 (2013).

[12] Clarke, Leon and Weyant, John and Birky, Alicia: On the sources of technological change: Assessing the evidence, Energy Economics, Elsevier, 28, 579-595 (2006).

[13] Belady, Christian L and Malone, Christopher G, Metrics and an infrastructure model to evaluate data center efficiency, Proceedings of the Pacific Rim/ASME International Electronic Packaging Technical Conference and Exhibition (IPACK), ASME, (2007).

[14] Rivoire, Suzanne and Shah, Mehul A and Ranganathan, Parthasarathy and Kozyrakis, Christos, JouleSort: a balanced energy-efficiency benchmark, Proceedings of the 2007 ACM SIGMOD international conference on Management of data, ACM, 365-376 (2007).

[15] Stanley, John R and Brill, Kenneth G and Koomey, Jonathan; Four Metrics Define Data Center Greenness Enabling Users to Quantify Energy Efficiency for Profit Initiatives, The Uptime Institute, Santa Fe, (2007).
[16] ENERGY, STAR; Report to congress on server and data center energy efficiency public law 109-431, Washington, D.C.: U.S. Environmental Protection Agency, Public law, 1, 109-431 (2007).

[17] Lu, Tao and Lü, Xiaoshu and Remes, Matias and Viljanen, Martti: Investigation of air management and energy performance in a data center in Finland: Case study, Energy and Buildings, Elsevier, 43, 3360-3372 (2011).

[18] Kant, Krishna; Data center evolution: A tutorial on state of the art, issues, and challenges; Computer Networks, Elsevier, 53, 2939-2965 (2009).

[19] Koomey, Jonathan G; Worldwide electricity used in data centers, Environmental Research Letters, IOP Publishing, 3, 034008 (2008).

[20] Hultman, Nathan E and Koomey, Jonathan G; The risk of surprise in energy technology costs; Environmental Research Letters; IOP Publishing, 2, 034002 (2007).

[21] Liu, Chunhui and Sia, Choon-Ling and Wei, Kwok-Kee, Adopting organizational virtualization in B2B firms: an empirical study in Singapore, Information \& management, Elsevier, 45, 429-437 (2008).

[22] Harris, Jason; Green Computing and Green IT: Best practices on regulations and industry initiatives, virtualization, power management, materials recycling and telecommuting, Lulu. com, (2008).

[23] Choi, Jeonghwan and Govindan, Sriram and Urgaonkar, Bhuvan and Sivasubramaniam, Anand; Profiling, prediction, and capping of power consumption in consolidated environments, Modeling, Analysis and Simulation of Computers and Telecommunication Systems, 2008. MASCOTS 2008. IEEE International Symposium on, IEEE, 1-10 (2008).

[24] Uddin, Mueen and Rahman, Azizah Abdul; Techniques to implement in green data centres to achieve energy efficiency and reduce global warming effects, International Journal of Global Warming, Inderscience, 3, 372-389 (2011).

[25] Sun, HS and Lee, SE; Case study of data centers energy performance, Energy and Buildings, Elsevier, 38, 522-533 (2006).

[26] Ricardo Lent, A model for network server performance and power consumption, Sustainable Computing: Informatics and Systems, http://www.sciencedirect.com/science/article/pii/S221053791200021, 3, 80-93 (2013).

[27] Rivoire, Suzanne and Ranganathan, Parthasarathy and Kozyrakis, Christos, A Comparison of High-Level Full-System Power Models, HotPower, 8, 3-3 (2008).

[28] Narayanan, Dushyanth; Power by proxy: a new approach to measurement, Microsoft Research Ltd., Cambridge, UK, (2002).

[29] Bertini, Luciano and Leite, Julius CB and Mossé, Daniel; Power optimization for dynamic configuration in heterogeneous web server clusters, Journal of Systems and Software, Elsevier, 83, 585-598 (2010).

[30] Kopparapu, Chandra; Load balancing servers, firewalls, and caches, John Wiley \& Sons, (2002).

[31] Stillwell, Mark and Schanzenbach, David and Vivien, Frédéric and Casanova, Henri; Resource allocation using virtual clusters, Cluster Computing and the Grid, 2009. CCGRID'09. 9th IEEE/ACM International Symposium on, IEEE, 260-267 (2009). 
[32] Patel, Chandrakant D; A vision of energy aware computing from chips to data centers, The International Symposium on Micro-Mechanical Engineering, (2003).

[33] Laudon, James; Performance/watt: the new server focus, ACM SIGARCH Computer Architecture News, ACM, 33, 5-13 (2005).

[34] Van den Bossche, Ruben and Vanmechelen, Kurt and Broeckhove, Jan; An evaluation of the benefits of finegrained value-based scheduling on general purpose clusters, Future Generation Computer Systems, Elsevier, 27, 1-9 (2011).

[35] AlEnawy, Tarek A and Aydin, Hakan; Energy-aware task allocation for rate monotonic scheduling, Real Time and Embedded Technology and Applications Symposium, 2005. RTAS 2005. 11th IEEE, IEEE, 213223 (2005).

[36] Yuan, Wanghong and Nahrstedt, Klara; Energyefficient soft real-time CPU scheduling for mobile multimedia systems, ACM SIGOPS Operating Systems Review, ACM, 149-163 (2003).

[37] Merkel, Andreas and Stoess, Jan and Bellosa, Frank; Resource-conscious scheduling for energy efficiency on multicore processors, Proceedings of the 5th European conference on Computer systems, ACM, 153-166 (2010).

[38] Mastroleon, Lykomidis and Bambos, Nicholas and Kozyrakis, Christos and Economou, Dimitris; Automatic power management schemes for Internet servers and data centers, Global Telecommunications Conference, 2005. GLOBECOM'05. IEEE, IEEE, 2, 5 (2005).

[39] Elnozahy, EN Mootaz and Kistler, Michael and Rajamony, Ramakrishnan; Energy-efficient server clusters, Lecture Notes in Computer Science, Springer, 2325, 179-197 (2003).

[40] Clidaras, Jimmy and Stiver, David W and Hamburgen, William and others; Water-based data center, Google Patents, (2009).

[41] Heath, Taliver and Diniz, Bruno and Carrera, Enrique $\mathrm{V}$ and Meira Jr, Wagner and Bianchini, Ricardo; Energy conservation in heterogeneous server clusters, Proceedings of the tenth ACM SIGPLAN symposium on Principles and practice of parallel programming, ACM, 186-195 (2005).

[42] Adachi, Motomitsu and Hiraoka, Takuro and Komatsu, Naohisa; A study on a resource allocation algorithm for on-demand data center services, Advanced Communication Technology, 2008. ICACT 2008. 10th International Conference on, IEEE, 1, 295-300 (2008).

[43] Petrucci, Vinicius and Loques, Orlando and Mossé, Daniel; A dynamic configuration model for powerefficient virtualized server clusters, 11th Brazillian Workshop on Real-Time and Embedded Systems (WTR), Citeseer, 2, (2009).

[44] Capit, Nicolas and Da Costa, Georges and Georgiou, Yiannis and Huard, Guillaume and Martin, Cyrille and Mounié, Grégory and Neyron, Pierre and Richard, Olivier; A batch scheduler with high level components, Cluster Computing and the Grid, 2005. CCGrid 2005. IEEE International Symposium on, IEEE, 2, 776-783 (2005).

[45] Bose, Ranjit and Luo, Xin; Integrative framework for assessing firms potential to undertake Green IT initiatives via virtualization-A theoretical perspective, The Journal of Strategic Information Systems, Elsevier, 20, 38-54 (2011).

[46] Belady, Christian L; In the data center, power and cooling costs more than the it equipment it supports, Electronics cooling, FLOMERICS LIMITED, 13, 24 (2007).

[47] Horvath, Tibor, Energy Management in Real-time Multi-tier Internet Services, University of Virginia, (2008).

[48] Urgaonkar, Bhuvan and Rosenberg, Arnold L and Shenoy, Prashant; Application placement on a cluster of servers, International Journal of Foundations of Computer Science, World Scientific, 18, 1023-1041 (2007).

[49] Ceuppens, Luc and Sardella, Alan and Kharitonov, Daniel; Power saving strategies and technologies in network equipment opportunities and challenges, risk and rewards; Applications and the Internet, 2008. SAINT 2008. International Symposium on, IEEE, 381384 (2008).

[50] Loper, Joe and Parr, Sara; Energy efficiency in data centers: A new policy frontier, Environmental Quality Management, Wiley Online Library, 16, 83-97 (2007).

[51] Belady, Christian and Rawson, Andy and Pfleuger, JOHN and Cader, TAHIR; Green grid data center power efficiency metrics: PUE and DCiE, the green grid, 1-9 (2008).

[52] Malik, R and Barthel, S; Energy Efficiency, its Benefits and Methods to close the Efficiency gap, Applied Power Electronics Conference and Exposition, 2008. APEC 2008. Twenty-Third Annual IEEE, IEEE, 417-422 (2008).

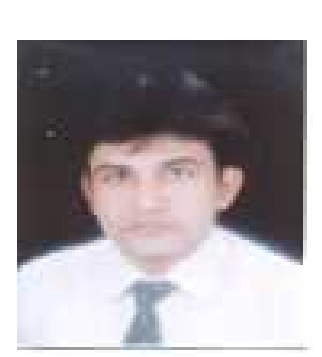

Mueen Uddin is a Postdoctoral Research Fellow at the International Islamic University Malaysia (IIUM). He received his $\mathrm{PhD}$ from Universiti Teknologi Malaysia (UTM) in 2012. His research interests include green IT, energy-efficient data centers, green metrics, global warming effects, virtualization, cloud computing, digital content protection and deep packet inspection, intrusion detection and prevention systems, and MANET routing protocols. Dr. Mueen earned his BS and MS in Computer Science from Isra University Pakistan with specialization in information networks. He has published more than 35 papers in indexed and reputable international journals. 


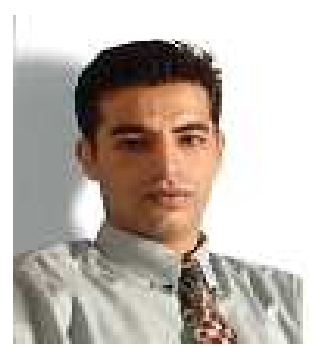

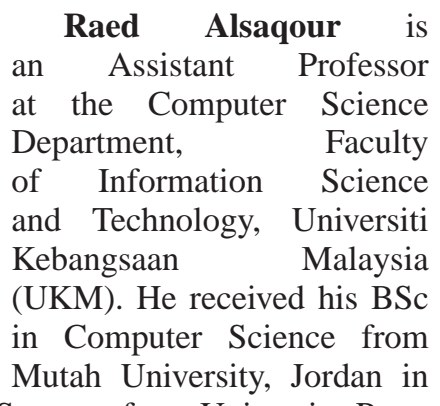
1997; MSc in Distributed Systems from University Putra Malaysia in 2003; and $\mathrm{PhD}$ in Wireless Communication Systems from UKM in 2008. His research interests include wireless, ad hoc, and vehicular networks; routing protocols; simulation; and network performance evaluation.

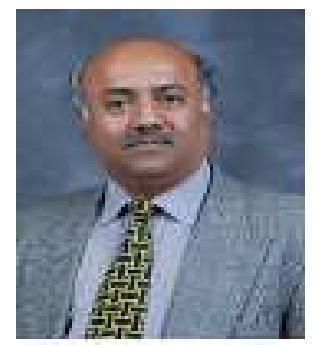

\section{Asadullah}

is a Professor at IIUM. His research interests include IS and IT systems management, multimedia systems, and compression techniques. Dr. Shah earned his $\mathrm{PhD}$ in Multimedia Systems from the University of Surrey, UK. He is a member of the Institute of Electrical and Electronic

Engineers (IEEE) and the Association for Computing Machinery (ACM). He is a renowned researcher with over 80 publications in journals and conferences.

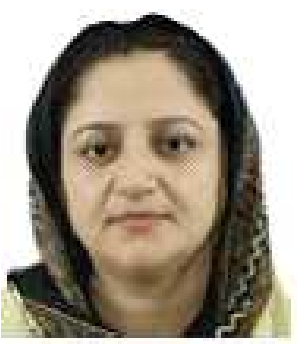

Tanzila Saba r earned
her PhD in Document
Information Security
and Management from
the Faculty of Computing,
UTM in 2012. She won the
Best Student Award from the
Faculty in 2012. Currently,
she is an Assistant Professor
at the College of Computer
and Information Sciences,

Prince Sultan University, Riyadh, KSA. Her more than 15 research papers are ISI/SCIE indexed. She is included in Marquis Whos Who (S\&T) 2012 list because of her excellent research achievements. 